\title{
Embryonic diapause in the European roe deer (Capreolus capreolus)
}

\author{
VA van der Weijden and SE Ulbrich \\ Institute of Agricultural Sciences, ETH Zurich, Switzerland
}

Corresponding author email: seu@ethz.ch

\begin{abstract}
The first written reports on embryonic diapause in the European roe deer (Capreolus capreolus) date back to 1843 and 1854 . While the rut takes place in July/August, rapid embryo elongation and implantation take place in December/January, after a five month period of embryonic diapause. The post-implantation development lasts another five months and an average of two fawns are born in May. As fertilization and subsequent implantation are decoupled, the roe deer offers the opportunity to study changes in hormones, embryonic and endometrial morphology and transcriptome dynamics, as well as the uterine microenvironment in a high time-dimension. So far, it has been shown that peripheral increases in prolactin, estradiol-17 $\beta$ and progesterone are a consequence, rather than the cause of reactivation of embryo development. Increased uterine secretions and the presence of embryonic cell organelles coincide with embryo elongation. This suggests that the "quiet embryo" hypothesis might hold for diapausing embryos. The factors controlling embryonic developmental pace in the roe deer are yet to be identified. We propose a multi-omics approach to determine the temporal molecular changes during diapause, prior to embryo elongation, which morphologically marks the reactivation of embryo development, and upon elongation. Thereby, we will obtain insights into the molecular mechanisms and environmental cues that inhibit and drive cell proliferation. By data integration, we aim at identifying the origin of the factors associated with reduced embryonic developmental pace. The knowledge of a species-independent mechanism driving embryo development past the blastocyst stage is of high interest to reproductive biologists and for species conservation programs.
\end{abstract}

Keywords: Embryonic diapause, European roe deer, embryo-maternal communication

\section{Introduction}

The phenomenon of embryonic diapause

Already in the $19^{\text {th }}$ century, the reproductive cycle of the roe deer (Capreolus capreolus) has been thoroughly described. Driven by observations from hunters, the first written reports on embryonic diapause in the European roe deer were published in 1843 and 1854 by Ziegler and Bischoff [1]. Mating was observed in July and August, but was thought to be a "false mating", as neither embryos nor fetuses were found in the uterus until late December. "True" mating was observed in December and was followed by implanted fetuses from January onwards, resulting in offspring being born in May/June [1]. Therefore, the theory of the "false" mating was introduced [1]. When Ziegler and Bischoff described the morphology of the ovaries, uterus and testicles, the "false" 
mating was refuted, as functional semen was not found in bucks in December and does only displayed fresh ovulations in August [1]. Thorough and very patient investigations of the uterine content with a magnification glass revealed the presence of embryos from the middle of August onwards [1]. Thus, the concept of an "embryonic diapause" was formed, which has been kept until today. Fifty years later, detailed morphological descriptions and illustrations of the developing embryo were published by Keibel in 1902 in Tübingen, Germany [2]. These early reports on embryo development and characteristics of the reproductive tract in roe deer have been reviewed in English by Short and Hay in 1966 [3]. Within the last decades of the $20^{\text {th }}$ century, a number of studies have emphasized on characterizing the hormonal environment, embryonic and endometrial morphological changes, and the uterine microenvironment to identify the mechanisms that control embryonic diapause in roe deer [3]. Only recently, a new attempt to unravel embryonic diapause in the roe deer was initiated using a novel multi-omics approach that includes a molecular perspective (own unpublished data) [4-6].

In the roe deer, the rut takes place in July/August, while rapid embryo elongation and implantation occur five months later in December/January [3]. This indicates the obligate period of embryonic diapause in this species [3]. The post-implantation development lasts another four to five months, and an average of two fawns (range 1-3) are born in May [3]. The high pregnancy rates in the roe deer suggest that reproduction is highly efficient. In an Italian roe deer population study, the average number of implanted fetuses in January and February was $1.4 \pm 0.1$ per doe [7]. A study with captive roe deer in France found 1.8 fawns per reproductive female [8], while a field study in Norway found that most does gave birth to two fawns [9]. Other studies determined the sex ratio and found that the female:male sex ratio was 1:1 [10], and twins were mostly nonhomozygous [3].

At present, embryonic diapause is known to occur in over 130 species across seven mammalian orders including Marsupialia, Insectivora, Chroptera, Edentata, Carnivora, Rodentia and Artiodactyla [11]. It was first described in roe deer and most recently in the panda [12]. Its occurrence is either obligate or facultative, the latter being driven by species-specific stimuli [11]. Species displaying obligate diapause include the tammar wallaby and mink, while the mouse displays a facultative diapause [11]. It is generally accepted that embryonic diapause is a strategy to deliver offspring in the most favorable season for survival [13-15]. It may also serve as strategy to rapidly overcome offspring losses like in Marsupialia [16], or it may be related to paternal fitness like in the roe deer [1]. Three distinct phases of embryonic diapause have been described, including arrest of cell division, maintenance of diapause and resumption of development [14]. The regulatory mechanisms known to date vary widely among Eutheria, and even within a family. Common to all species displaying delayed implantation is that the uterine microenvironment supports embryo survival for a prolonged period of time without negatively affecting it [11]. Yet, the uterine microenvironment is hypothesized to lack stimulatory factors to initiate resumption of development [13], and/or to contain inhibitory factors for maintenance of diapause.

Other mammalian species that display diapause show a distinct presence of factors implicated in the regulation of diapause. The hormonal uterine stimulation that causes resumption of embryo development has been shown to be species-specific, while the embryonic molecular factors seem conserved [11]. In mice, diapause can be induced by lactation, where the high PRL levels repress $E 2$, or by ovariectomy resulting in low peripheral E2 levels. Removal of the suckling pups or an E2 injection causes resumption of embryo development. In the tammar wallaby, there is a surge of both E2 and P4, while in mink, prolactin and P4 cause resumption of embryo development [11]. The embryonic molecular factors involved in resumption of embryo development seem conserved and have been reviewed by Renfree and Fenelon [11]. 
In the roe deer, hormone changes, morphological descriptions and a first characterization of the uterine microenvironment have provided insights into the pre-implantation developmental dynamics. Since then, several research attempts have been made to unravel the phenomenon of embryonic diapause in roe deer. More than 150 years after its discovery, the factors controlling embryonic developmental pace in the roe deer still remain to be identified.

\section{Pre-implantation developmental dynamics in roe deer}

\section{Hormones}

The roe deer displays seasonal ovulation controlled by melatonin [17]. An experimentally increased peripheral melatonin level caused a sudden drop in prolactin (PRL), shifting ovulation to 72 days earlier [17]. In this experiment, does received a melatonin implant on the $16^{\text {th }}$ of April and hereafter monthly until August [17]. Within 6 days after implant insertion, the PRL concentrations decreased and remained low until August [17]. This shifted the ovulation period to be 72 days earlier compared to non-treated control animals [17]. Likewise, ovulation was delayed by 46 days by prolonged exposure to days with long light periods [17]. In the latter experiment, animals were exposed to a 16 hours light and 8 hours dark regime directly after the summer solstice [17]. Thereupon, the first increase of progesterone (P4) was delayed by 46 days, which was found to be the result of ovulation on the $14^{\text {th }}$ of October versus the $28^{\text {th }}$ of August in control animals [17].

A luteinizing hormone (LH) peak was shown to precede ovulation, followed by elevated luteal P4 production [3, 18, 19]. In both pregnant and non-pregnant animals, P4 increased between July and August [20]. A second increase in P4 has been observed in pregnant animals in January [20]. From January onwards, the circulating P4 were one order of magnitude higher in pregnant versus nonpregnant animals [20]. In non-pregnant animals, P4 decreased in April and reached basal levels in May, while in pregnant animals, P4 gradually decreased from March to May [20]. In June and July, P4 remained low and increased again after ovulation in August [20].

After ovulation, does develop on average two corpora lutea $(C L)[4,18]$. Their weight did not change over the course of pregnancy and the CL did not degenerate after embryo implantation [3]. The number of CL correlated neither with peripheral, nor uterine P4 levels [4]. Based on its size, histological appearance and $\mathrm{P} 4$ content, the $\mathrm{CL}$ remained fully functional for about five months, irrespective of the pregnancy status $[3,21]$. A decrease in the number of tertiary follicles from October to January has been determined [4].

The luteal oxytocin (OXT) concentration, as determined by a radioimmunoassay on luteal homogenates, was neither related to the number of ovulations, nor to the time of the year [22]. Unlike in other ungulates, an OXT-induced prostaglandin F2 $\alpha$ (PGF2 $\alpha$ ) secretion was neither observed on day 3 after induced estrus, nor in late October in animals exposed to long days [22]. Nevertheless, intramuscular prostaglandin injections in October caused a rapid decrease in P4 and induced an LH peak 4 days after the injections [18]. Like during a natural ovulation, the LH peak preceded a rise in $\mathrm{P} 4$ [18]. This indicates that the $\mathrm{CL}$ is principally sensitive to the luteolytic effect of prostaglandins. However, the lack of an OXT increase most likely explains why the roe deer is a mono-estrous species. Peripheral PRL in roe deer plasma samples obtained from huntings between the $9^{\text {th }}$ of November and $28^{\text {th }}$ of January were assessed to determine its role in resumption of embryo development $[23,24]$. As PRL remained basal during diapause, but increased after implantation, it has been suggested that PRL does not play a role in resumption of embryo development $[23,24]$. The peripheral and local uterine concentrations of P4, estradiol$17 \beta$ (E2) and total estrogens remained unchanged during diapause [4], but increased after implantation, suggesting at least a partial contribution of the developing placenta $[20,23,25-27]$. 
Thus, the increases in PRL, E2 and P4 with fetal development are thought to be a consequence rather than the cause of reactivation of embryo development.

\section{Reproductive tract - uterus}

In roe deer, the uterus consists of two horns that are joined by connective tissue [1, 3]. Each horn has four non-glandular caruncular ridges that form the later site of placentation. Placentation is initiated by the formation of crypts at the time of embryo elongation [3, 21]. The intercaruncular endometrium is rich in glands and the opening of the glands are enlarged at embryo elongation [3]. The height of the endometrial epithelial cells was associated with the drop of E2 after estrus during the breeding season [21]. The glandular epithelium has been described as rich in vesicles from the Golgi apparatus during diapause, which disappeared during elongation $[25,28]$. This is in line with a reported increase in uterine protein synthesis with developmental progression [24], and suggests a maternal contribution to embryo elongation.

\section{Embryo}

During diapause, the embryo has hatched from the zona pellucida, resides in the uterus and its rapid elongation marks the end of diapause [3]. Whether hatching takes place in the oviduct or uterus remains unknown. In vivo flushing experiments on day 13 of development have suggested that embryos start hatching in the uterus on day 13 (Unpublished data; Drews et al. 2019 Gliding into diapause: early embryo development in roe deer). The embryos display an increase in size with age [3]. The BrdU incorporation in trophoblast cells as measure for cell proliferation increased from $5 \%$ in October/November to $10 \%$ in November/December [29], and there was a 22.5 -fold higher protein incorporation at elongation versus diapause [23, 24]. By numerous field sample collections, our group substantiated earlier reports [1-3, 25, 28, 29] of a continuous increase in embryonic size with time (Figure 1) (Unpublished data; Rüegg et al. 2019 Trophectoderm and Embryoblast of the European Roe Deer (Capreolus capreolus) both proliferate at slow pace during Embryonic Diapause) [4-6]. In. brief, the reproductive tract of animals was obtained from authorized regular huntings between September and January. The embryos were collected by uterine flushing, and the embryos were microscopically visualized and their diameter was determined. Over the four sampling seasons 2015-2018, a comparable growth pattern was observed. Embryo elongation starts approximately at $4 \mathrm{~mm}$. Prior to elongation, there seems to be a log-linear increase of the size of the embryo with time. After resumption of development, the embryos rapidly increase in size and elongate.

The embryonic morphology has been characterized using scanning and transmission electron microscopy by Aitken in 1973 and 1975 [25]. During diapause, the embryos displayed branched microvilli on the surface of the trophoblast [25] and contained many electron-dense droplets [28]. Most embryonic cells were largely devoid of organelles, although mitochondria and poorly developed endoplasmic reticulum have been reported [28]. With developmental progression, there was a decrease in electron-dense droplets and elongation was characterized by the presence of cell organelles, suggesting a higher proliferation rate and increased metabolic demands [28]. 

69

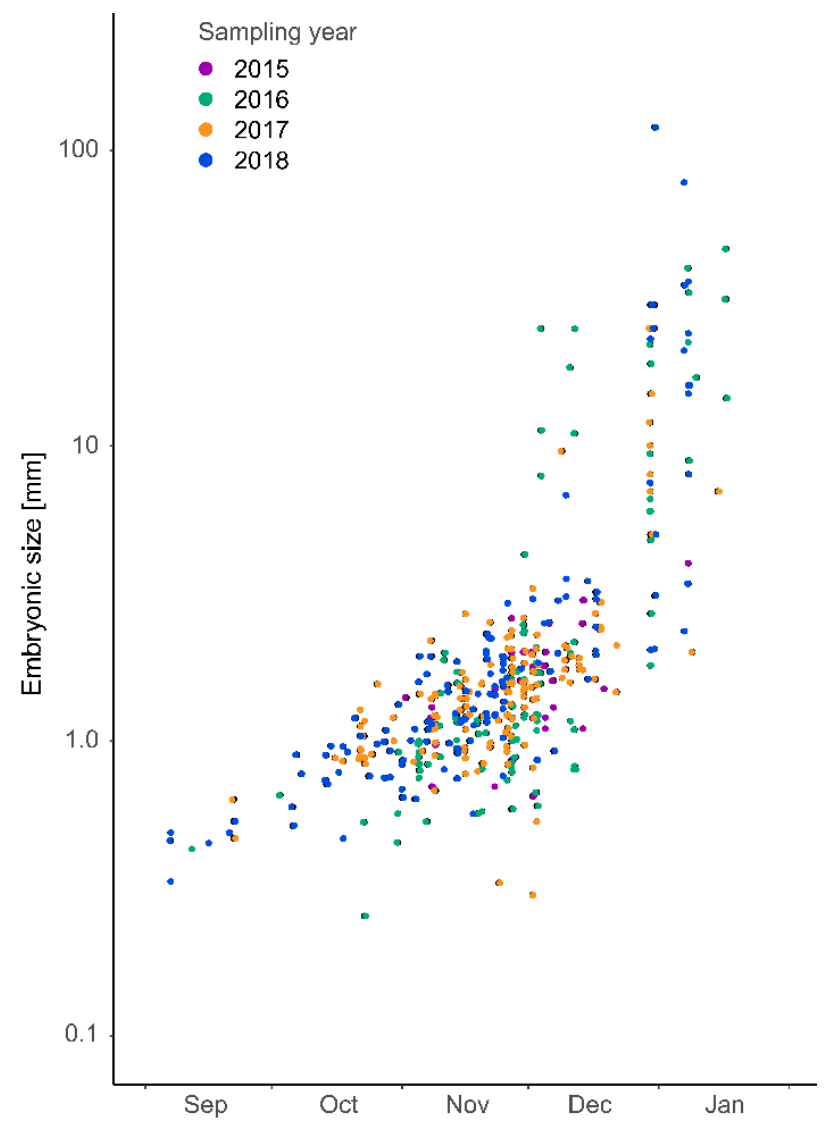

Figure 1. Embryonic growth during diapause (prior to elongation) and after resumption of embryo development. Different sampling years are indicated with different colors.

While the embryos were not found to secrete interferon tau (IFNT) prior to implantation [6, 22], the embryos secreted roe deer-specific pregnancy associated glycoproteins (PAGs) upon implantation [26]. An interspecies embryo transfer experiment with mice, a species with facultative diapause, and sheep, a species without diapause, showed that the mouse uterine environment induced diapause in sheep embryos [15]. Therefore, the uterine microenvironment is considered important for embryo survival and can affect embryonic developmental pace. An interspecies embryo transfer experiment, where roe deer embryos were transferred into sheep, showed that roe deer embryos failed to secrete species-specific signals required to overcome cyclicity in sheep [3]. A comparable interspecies embryo transfer experiment of roe deer embryos to cattle likewise failed (own unpublished data). This suggests that species-specific signals are required for successful establishment of pregnancy.

\section{Uterine microenvironment}

The mammalian uterine microenvironment, comprising the uterine fluid, constantly changes during early embryo development to support its survival and for the establishment of pregnancy. The uterine fluid presents a mixture of signals from embryonic and endometrial origin, and contains proteins, amino acids (AA), nutrients, ions and metabolites [30-32]. To understand the embryonic metabolic needs, in vitro culture systems have been used for bovine, porcine and human embryos [33-37]. Additionally, it has been shown that glucose and pyruvate uptake increases in reactivated mouse embryos [38]. The metabolism of developing embryos versus nonviable embryos that display developmental arrest has been studied. This resulted in the postulation of the "quiet embryo" hypothesis, where Leese et al. proposed that viable embryos 
have a "quieter" metabolism than embryos displaying developmental arrest $[39,40]$. This is of particular interest, as diapausing embryos can be regarded as "quiet". They display a low developmental pace, yet regain developmental competence upon reactivation. The identification of factors crucial for keeping embryos at a low developmental pace and metabolic rates could improve current in vitro fertilization strategies.

In diapausing species, the uterine microenvironment has been hypothesized to temporarily lack factors that induce and support the process of embryonic growth [25]. The uterine secretions in roe deer increased by $150 \%$ between maintenance of diapause and resumption of development [23]. Aditionally, qualitative changes in secreted proteins were evident at implantation [24]. Embryo elongation correlated with a rise in total hexose, fructose, total protein, $\alpha$-amino nitrogen and calcium $[25,41,42]$. We have previously shown a high presence of proteins involved in cellular detoxification during diapause, while resumption of development was characterized by the presence of proliferation-inducing proteins [5]. Neither changes in uterine fluid zinc, glucose nor PGF2 $\alpha$ were found to coincide with embryo elongation $[25,41]$. The changes in the roe deer uterine microenvironment constituents suggest reactivation via environmental cues that remain to be defined.

\section{Key factors regulating embryonic diapause in the roe deer}

Although continues effort has been made to unravel the regulation of diapause in the roe deer, research in this area had ceased at the beginning of the $21^{\text {st }}$ century. The recent technological advances in the field of multi-omics, including transcriptome, proteome and metabolome analyses, now offer the opportunity to identify key factors involved in the regulation of embryonic diapause. As in other diapausing species, it is not known if developmental arrest is a developmental program inherent to the embryo or whether it is regulated by maternal signals, which are influenced by external factors such as a variation in photoperiod or feed availability. Thus, this requires research to specifically aim at identifying species-specific reactivation signals as well as conserved embryonic factors. First studies on the uterine microenvironment proteome have shown a decrease of proteins involved in cellular detoxification and polyamine metabolism after resumption of embryo development, while proteins involved in the induction of proliferation where higher abundant after resumption of embryo development than during diapause [5].

\section{Outlook}

Diapausing animals are ideal model species to investigate the factors that accumulate in the uterine microenvironment. In diapausing species, specific factors influencing embryonic growth are hypothesized to differ between developmental arrest and reactivation. They could be better detected by comparing the embryo, endometrium and uterine microenvironment at different stages of development (Figure 2). It is deemed feasible to unravel the temporal molecular changes during diapause, prior to embryo elongation, which marks the reactivation of embryo development, and upon elongation. Thereby, insight into the molecular mechanisms and environmental cues that inhibit and drive cell proliferation will be otained. By integration of the multi-omics data, the origin of the factors associated with changes in developmental pace of the embryos will be identified. 


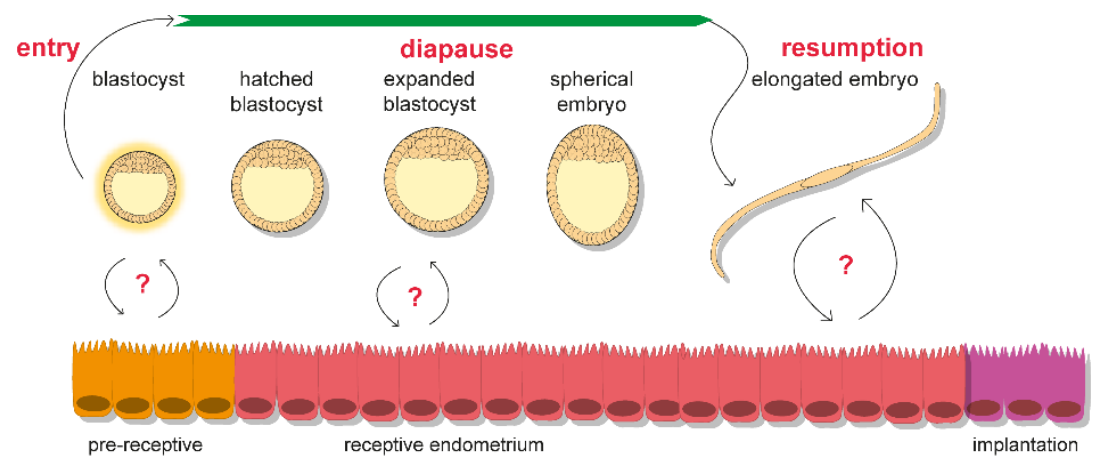

embryos
- time-course transcriptomics
by RNA-seq
uterine fluid
- proteomics by LC-MS/MS
- amino acids and acylcarnitines
quantification by targeted LC-MS/MS
Iuminal epithelium
- time-course transcriptomics
by RNA-seq

Figure 2. Proposed multi-omics approach to identify key factors in the regulation of embryonic diapause.

Species displaying diapause represent an ideal model to investigate the factors involved in embryonic developmental pace regulation. In non-diapausing species, signals for further development after the blastocyst stage are continuous. Therefore, the autonomous embryo development and changes in the uterine microenvironment composition are difficult to intercept. The time-window in which an additional signal to further embryonic development is needed is prolonged in diapausing species. Hence, the European roe deer, displaying similar developmental characteristics as other non-diapausing ruminant species, is an interesting animal model.

Identified factors for diapause regulation may allow the control of developmental pace in vitro. This would enhance chances for successful implantation after transfer, due to the achievement of a stable embryo condition, i.e., a "quiet embryo", and to an increased robustness of the delayed embryo, specifically in terms of coping with an only sub-optimally synchronized maternal endometrium.

Since the embryo-maternal dialogue is mediated by the uterine microenvironment, knowledge about its composition in the pre-implantation period is crucial. The knowledge of a speciesindependent mechanism driving embryo development past the blastocysts stage would be of high interest to reproductive biologists in human and veterinary medicine, but also for species conservation programs, as it will shed light on the pregnancy support and key factors for developing post-fertilization contraceptives.

\section{Declaration of interest}

The authors declare that there are no conflicts of interest.

\section{Funding}

This study was funded by the Swiss National Science Foundation SNSF (31003A_159734 and 310030_185026).

\section{Acknowledgements}

We are thankful to all Diapause conference participants for the vivid discussions. The authors are active participants of the COST Action CA16119 (CellFit - In vitro 3D total cell guidance and fitness). 


\section{References}

[1] Bischoff TLW. 1854 Die Entwicklungsgeschichte des Rehes. Giessen, J. Bicker'sche Buchhandlung.

[2] Keibel F. 1902 Die Entwicklung des Rehes bis zur Anlage des Mesoblast. Anat Physiol Suppl 292.

[3] Short RV and Hay MF. 1966 Delayed Implantation in the roe deer Capreolus capreolus, in Comparative Biology of Reproduction in Mammals, I.W. Rowlands, Editor. Academic Press: New York. 173194.

[4] Drews B, Rudolf Vegas A, van der Weijden VA, Milojevic V, Hankele AK, Schuler G and Ulbrich SE. 2019 Do ovarian steroid hormones control the resumption of embryonic growth following the period of diapause in roe deer (Capreolus capreolus)? Reprod Biol. 19(2) 149-157.

[5] van der Weijden VA, Bick J, Bauersachs S, Arnold GJ, Frohlich T, Drews B and Ulbrich SE. 2019 Uterine fluid proteome changes during diapause and resumption of embryo development in roe deer. Reproduction 158(1) 13-24.

[6] van der Weijden VA, Puntar B, Rudolf Vegas A, Milojevic V, Schanzenbach Cl, Kowalewski MP, Drews B and Ulbrich SE. 2019 Endometrial luminal epithelial cells sense embryo elongation in the roe deer independent of interferon-tau. Biol Reprod 101(5) 882-892.

[7] Focardi S, Pelliccioni E, Petrucco R and Toso S. 2002 Spatial patterns and density dependence in the dynamics of a roe deer (Capreolus capreolus) population in central Italy. Oecologia 130(3) 411-419.

[8] Gaillard JM, Delorme D and Jullien JM. 1993 Effects of cohort, sex, and birth date on body development of roe deer (Capreolus capreolus) fawns. Oecologia 94(1) 57-61.

[9] Andersen R and Linnell JD. 1996 Variation in maternal investment in a small cervid; the effects of cohort, sex, litter size and time of birth in roe deer (Capreolus capreolus) fawns. Oecologia 109(1) 74-79.

[10] Aitken RJ. 1974 Sex chromatin formation in the blastocyst of the roe deer (Capreolus Capreolus) during delayed implantation. J Reprod Fertil 40(1) 235-9.

[11] Renfree MB and Fenelon JC. 2017 The enigma of embryonic diapause. Development 144(18) 31993210.

[12] Fenelon JC and Renfree MB. 2018 The history of the discovery of embryonic diapause in mammals. Biology of Reproduction 99(1) 242-251.

[13] Mead RA. 1993 Embryonic diapause in vertebrates. J Exp Zool 266(6) 629-41.

[14] Renfree MB and Shaw G. 2000 Diapause. Annu Rev Physiol 62 353-75.

[15] Ptak GE, Tacconi E, Czernik M, Toschi P, Modlinski JA and Loi P. 2012 Embryonic diapause is conserved across mammals. PLoS One 7(3) e33027.

[16] Renfree MB and Shaw G. 2014 Embryo-endometrial interactions during early development after embryonic diapause in the marsupial tammar wallaby. Int J Dev Biol 58(2-4) 175-81.

[17] Sempere AJ, Blanvillain C, Mauget R, Lacroix A and Chemineau P. 1995. Effects of melatonin implantation or artificial long days on seasonal ovulatory activity in roe deer (Capreolus capreolus L.). Animal Reproduction Science 38 127-136.

[18] Sempere AJ, Mauget R and Chemineau P. 1992 Experimental induction of luteal cyclicity in roe deer (Capreolus capreolus). J Reprod Fertil 96(1) 379-84.

[19] Schams D, Barth D and Karg H. 1980 LH, FSH and progesterone concentrations in peripheral plasma of the female roe deer (Capreolus capreolus) during the rutting season. J Reprod Fertil 60(1) 109-14.

[20] Hoffmann B, Barth D and Karg H. 1978 Progesterone and estrogen levels in peripheral plasma of the pregnant and nonpregnant roe deer (Capreolus capreolus). Biol Reprod 19(5) 931-5.

[21] Aitken RJ, Burton J, Hawkins J, Kerr-Wilson R, Short RV and Steven DH. 1973 Histological and ultrastructural changes in the blastocyst and reproductive tract of the roe deer, Capreolus capreolus, during delayed implantation. J Reprod Fertil 34(3) 481-93.

[22] Flint AP, Krzywinski A, Sempere AJ, Mauget R and Lacroix A. 1994 Luteal oxytocin and monoestry in the roe deer Capreolus capreolus. J Reprod Fertil 101(3) 651-6.

[23] Lambert RT. 1999 Conceptus-endometrial interactions and reproductive hormone profiles during 
embryonic diapause and reactivation of the blastocyst in the European roe deer (Capreolus capreolus). Rangifer 19(1) 41.

[24] Lambert RT, Ashworth CJ, Beattie L, Gebbie FE, Hutchinson JS, Kyle DJ and Racey PA. 2001 Temporal changes in reproductive hormones and conceptus-endometrial interactions during embryonic diapause and reactivation of the blastocyst in European roe deer (Capreolus capreolus). Reproduction 121(6) 863-71.

[25] Aitken RJ. 1974 Delayed implantation in roe deer (Capreolus capreolus). J Reprod Fertil 39(1) 225-

33.

[26] Lambert RT. 2005 A Pregnancy-Associated Glycoprotein (Pag) Unique to the Roe Deer (Capreolus Capreolus) and Its Role in the Termination of Embryonic Diapause and Maternal Recognition of Pregnancy. Israel Journal of Zoology 51(1) 1-11.

[27] Sempere AJ, Renaud G and Bariteau F. 1989 Embryonic Development Measured by Ultrasonography and Plasma Progesterone Concentrations in Roe Deer (Capreolus capreolus L.). Animal Reproduction Science 20 155-164.

[28] Aitken RJ. 1975 Ultrastructure of the blastocyst and endometrium of the roe deer (Capreolus capreolus) during delayed implantation. J Anat 119(Pt 2) 369-84.

[29] Lengwinat T and Meyer HH. 1996 Investigations of BrdU incorporation in roe deer blastocysts in vitro. Anim Reprod Sci 45(1-2) 103-7.

[30] Gao H, Wu G, Spencer TE, Johnson GA, Li X and Bazer FW. 2009 Select nutrients in the ovine uterine lumen. I. Amino acids, glucose, and ions in uterine lumenal flushings of cyclic and pregnant ewes. Biol Reprod 80(1) 86-93.

[31] Harris SE, Gopichandran N, Picton HM, Leese HJ and Orsi NM. 2005 Nutrient concentrations in murine follicular fluid and the female reproductive tract. Theriogenology 64(4) 992-1006.

[32] Munoz M, Corrales FJ, Caamano JN, Diez C, Trigal B, Mora MI, Martin D, Carrocera S and Gomez E. 2012 Proteome of the early embryo-maternal dialogue in the cattle uterus. J Proteome Res 11(2) 751-66.

[33] Guerif F, McKeegan P, Leese HJ and Sturmey RG. 2013 A simple approach for COnsumption and RElease (CORE) analysis of metabolic activity in single mammalian embryos. PLoS One 8(8) e67834.

[34] Thompson JG, Partridge RJ, Houghton FD, Cox Cl and Leese HJ. 1996 Oxygen uptake and carbohydrate metabolism by in vitro derived bovine embryos. J Reprod Fertil 106(2) 299-306.

[35] Sturmey RG and Leese HJ. 2003 Energy metabolism in pig oocytes and early embryos. Reproduction 126(2) 197-204.

[36] Leese HJ, Conaghan J, Martin KL and Hardy K. 1993 Early human embryo metabolism. Bioessays 15(4) 259-64.

[37] Conaghan J, Handyside AH, Winston RM and Leese HJ. 1993 Effects of pyruvate and glucose on the development of human preimplantation embryos in vitro. J Reprod Fertil 99(1) 87-95.

[38] Spindler RE, Renfree MB and Gardner DK. 1996 Carbohydrate uptake by quiescent and reactivated mouse blastocysts. J Exp Zool 276(2) 132-7.

[39] Leese HJ. 2002 Quiet please, do not disturb: a hypothesis of embryo metabolism and viability. Bioessays 24(9) 845-9.

[40] Leese HJ, Baumann CG, Brison DR, McEvoy TG and Sturmey RG. 2008 Metabolism of the viable mammalian embryo: quietness revisited. Mol Hum Reprod 14(12) 667-72.

[41] Aitken RJ. 1974 Calcium and zinc in the endometrium and uterine flushings of the roe deer (Capreolus capreolus) during delayed implantation. J Reprod Fertil 40(2) 333-40.

[42] Aitken RJ. 1976. Uterine secretion of fructose in the roe deer. J Reprod Fertil 46(2) 439-40.

\section{Audience Discussion}

Grazyna Ptak: Is the development of the roe deer really slow and did you try to compare it to other deer which are not diapausing, like fallow deer or red deer, in order to be really sure? Because maybe cow embryos are not the perfect control in this case? 
Susanne Ulbrich: You're right, not much is known about other species, the fallow deer would be the most likely one to be easily studied, we like this idea as well. What we think from the cow is that implantation would also be slow, but yet nobody says the embryo of the cow has a diapause, simply it implants only 18 days after fertilization. So, "slow" I would put in parenthesis, but what is slow? Yes, slower than other species which are well characterized, but, good point, it might not be different from other species.

Jane Fenelon: Do you know when embryonic genome activation occurs in the roe deer?

Susanne Ulbrich: Our data is very scarce in this very early development in vivo. So, we don't know when fertilization exactly takes place. From all the hunting we have no idea how old the embryos are. This is partly why we think our in vitro culture/production is so important, because that would be one of the questions we want to answer.

Jane Fenelon: Do you think the slow delay of the roe deer in culture is partly due to the culture medium, because the embryo didn't look overly happy.

Susanne Ulbrich: True, yes, we don't know. So, the only indication I have in assuming that that might be not due to the in vitro culture is the in vivo findings, We hope if we molecularly characterize the developing embryos in vitro, we can discover whether or not they are parthenogenetic and whether the sort of pace they take regarding their gene expression is like other species.

Jane Fenelon: Do you think there are distinct stages in the diapause in term of early, mid and late diapause, or is it really just a slowing down of the development as opposed to 'this is diapause'?

Susanne Ulbrich: In the very early phase of diapause, we can't get samples from hunting, because the hunting season isn't open and we simply don't know whether they mated or not. So, this is a difficulty regarding our sampling possibilities. We will do the characterization, so for me it's very interesting to know the in vitro culture, because I have thought that the embryo, upon entering into the uterus, escaping from the zona, would then be inhibited by growth and thereby this would determine the beginning of diapause, We were very excited and surprised to see that the embryo would enter the uterus in an earlier stage than we had expected. That would then imply that it might be slow from the beginning on, but yet not so slow as you would have it during diapause. During that diapause we hope to distinguish these different phases. We'll wait for Vera (van der Weijden) to show this.

Susanne Ulbrich: Currently the only thing we can do is characterize morphology regarding the size. Then we extracted the DNA and could characterize the cell count and we will see later that the cell count between the inner cell mass and the trophectoderm on the one hand is obvious and on the other hand difficult. Then the question whether we have distinct phases or whether we can take it as a continuous development is one of the technical approaches or the conceptual approaches, we do this in trying to mirror both sides, the embryonic side, the uterine side and the histotrop (everybody says histotroph but I like to emphasize the difference between histotroph and histotrop), so the uterine flushing as a substrate, that has tropic and trophic effects and thereby I think that we hopefully can deduce downwards the factors that then induce the morphological changes, so that then actually your point, namely that we're looking for something that we don't know yet, will arise.

Katarina Jewgenow: Other than P4 and PR which you reported upon, did you think about using antigestagens to block the hormone action of progesterone during diapause?

Susanne Ulbrich: There are earlier studies which followed individual roe deer across the season and this is something we want to still also apply. Other than the gestagen progesterone, I'm very interested and this is also something Anna Hankele did. She set up an assay for neurogestagens, so 
5-alpha and 3-alpha-reduced and 20-alpha-reduced metabolites of gestagens, that could also take part in this concerted action of whatever the $\mathrm{CL}$ is synthesizing other than progesterone. We will first characterize the spectrum of other gestagens and second, because experimentally it is more difficult, we also hope to apply antigestagens or even to induce luteolysis to see whether this obligatory phase of diapause is really needed or whether we can transfer an embryo later and then have a shortened diapause period.

Bruce Murphy: I guess some of what it shows is a little bit expected, the cell cycle is affected, but the difference between this and other diapause species is, it seems like a continuum of action, so it's not really suppression of activity it's more like a complete smothering of the activity and that's harder to explain than on and off, turning the lights off partway is harder to explain. So can you do that?

Vera van der Weijden: It's quite apparent from the data that we have, that the changes are transient, so I guess if there were to be an inhibitor signal or an activating signal, we'd still be able to probably to pick that up after reactivation occurred. I think with the different levels of information we have, we're getting close. I think it's crucial to find that out first also based on the data we have and to then again look at what changes occur around this period, so there's not a clear on/off signal. I think with a change in abundance of multiple factors and then we'll be able to identify what inhibits or drive in the end the activation.

Katarina Jewgenow: I actually have a question, because there was a suggestion to shift the diapause and reactivation by embryo transfer, just taking out the blastocysts in August and then re-transferring them in winter. Do you think that the embryo has go through the (slowed down) growth phase before it can implant?

Marilyn Renfree: I want to know, what's the longest a roe deer can stay in diapause? Has anybody extended it artificially? The reason I ask is this and I know while the roe deer keeps growing, the tammar wallaby doesn't, and if we lutectomize or ovariectomise we can actually keep that blastocyst viable for two years. If we then give progesterone injection to 'replace' the $\mathrm{CL}$ it reactivates perfectly normally. In the case of the roe deer, how important is that elongation and how long can you push it out? Did anybody ever do ovariectomy - I don't remember if John Aitken did this years ago, when he was working on roe deer, but I think it could be quite informative for the sort of questions you are asking.

Susanne Ulbrich: Ovariectomy hasn't been done as far as I know

Marilyn Renfree: I saw 360 roe deer were included in your study and 537 preimplantation blastocysts, so do they typically come in twos?

Vera van der Weijden: Mostly we find on average $2 \mathrm{CL}$ per animal and with these 530ish embryos we have a recovery rate of around $80 \%$, so we find $80 \%$ of the embryos where we know ovulation has taken place and also from field observations from hunting they know that on average there are around 2 live births per animal. 